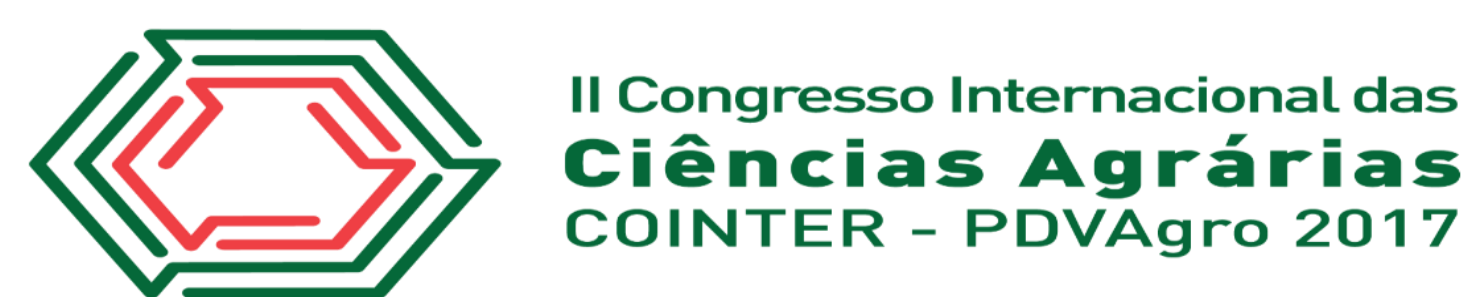

\title{
DIPTEROFAUNA ASSOCIADA A PLANTIOS COMERCIAIS DE COCO (Cocos nucifera L.) NO NORDESTE PARAENSE
}

Fernanda Valente Penner ${ }^{1}$; Lucas Faro Bastos ${ }^{2}$; Natalia Cristina de Almeida Azevedo ${ }^{3}$; Paulo Manoel Pontes Lins ${ }^{4}$; Telma Fátima Vieira Batista ${ }^{5}$

\section{Introdução}

Algumas ordens de insetos são excelentes indicadores de qualidade ambiental, porque desempenham funções variadas que permitem a manutenção do equilíbrio de ecossistemas terrestres.

Os insetos formam o mais numeroso e abundante grupo de seres vivos presentes no globo terrestre. Devido a sua reprodução acelerada e seu tamanho reduzido este grupo de animais ganhou predominância na maior parte dos ecossistemas existentes. São responsáveis por realizar atividades das mais valiosas possíveis para os seres humanos. Alguns podem ser extremamente prejudiciais, como pragas de culturas de grande valor econômico. Já outros desempenham funções de extrema relevância para a manutenção do equilíbrio ambiental.

Nessa perspectiva, o presente trabalho foi desenvolvido com o objetivo de prospectar a dipterofauna aérea em área comercial de coco no nordeste paraense, através da análise faunística das principais famílias encontradas.

\section{Fundamentação Teórica}

A família Arecaceae destaca se em muitos países por desempenhar importantes funções socioeconômicas principalmente em regiões tropicais, devido ao grande número de produtos que podem ser obtidos através de suas partes como estipes, folhas, raízes e principalmente através de seus frutos.

\footnotetext{
${ }^{1}$ Graduanda em Engenharia Florestal, Universidade Federal Rural da Amazônia, fernandavpenner@gmail.com

${ }^{2}$ Mestrando em Agronomia, Universidade Federal Rural da Amazônia, lucas.fbufra@gmail.com

${ }^{3}$ Graduanda em Engenharia Florestal, Universidade Federal Rural da Amazônia, naty.azeved@gmail.com

${ }^{4}$ Doutor em Ciências Agrárias, Gerência de pesquisas da Empresa Sococo Agroindústrias da Amazônia -Santa Isabel do Pará-Pa, paulom@sococo.com.br

${ }^{5}$ Doutora em Ciências Agrárias, Universidade Federal Rural da Amazônia, telma.batista@ufra.edu.br
} 
O coqueiro é um excelente exemplo de palmeira desta família, pois oferece um crescente potencial econômico nas principais regiões produtoras do Brasil, possuindo elevada produção durante o ano (Cavalcante, 2015).

A ordem Diptera constitui uma das maiores ordens dentre as cinco principais e mais numerosas do filo insecta (Coleóptera, Hemíptera, Díptera, Himenóptera e Lepidóptera). Sua grande maioria é compreendida por insetos de corpo frágil de tamanho reduzido e alguns deles minúsculos. Possuem elevada importância econômica e do ponto de vista agronômico cumprem funções imprescindíveis, como a polinização de vegetais. Alguns dípteros são detritívoros bastante úteis, ajudam na decomposição da matéria orgânica. Outros atuam como predadores ou parasitóides de diversas pragas. Além de atacar algumas espécies de plantas daninhas (Gullan \& Cranston, 2017).

Existem mais de 500 espécies de insetos relacionados à cultura do coqueiro, muitos destes indivíduos podem causar danos irreparáveis que reduzem o vigor das plantas e consequentemente causam o declínio da produção. Portanto técnicas de manejo integrado de pragas são uma excelente alternativa para mitigar o dano causado pelas pragas. E a ação de alguns insetos desta ordem como Winthemia analis (Macquart) (Tachinidae), que são parasitóides de pupas ou de larvas de Bassollis sophorae (L.) (Marcicano et al, 2009) são de extrema importância.

\section{Metodologia}

A pesquisa foi realizada em áreas comerciais da empresa Sococo $\left(01^{\circ} 13^{\prime} 40,16^{\prime \prime} \mathrm{S}\right.$ e $48^{\circ} 02^{\prime}$ 54,35” O), localizada no município de Santa Isabel do Pará-Pa. Conforme Köppen-Geiger a região possui classificação climática do tipo Afi, caracterizada principalmente por intensa precipitação. (SEMA, 2017).

As coletas biológicas (insetos) ocorreram com o uso de 40 armadilhas do tipo adesivas de coloração amarelas quadriculada, da marca ISCA, com dimensões em torno de $23 \times 28 \mathrm{~cm}$, alocadas nas copas dos coqueiros, onde permaneceram por 7 dias. A parcela experimental tinha 8,08 ha, com espaçamento de cultura de 7x7 m e o espaçamento utilizado para a disposição das armadilhas foi de 70 m entre uma linha de cultivo e $21 \mathrm{~m}$ entre plantas. No Laboratório de Proteção de Plantas UFRA foram realizados a triagem, quantificação e identificação taxonômica até ordem e família, com o uso de chaves dicotômicas. Espécimes prejudicados pelo estado de conservação foram identificados apenas a ordem, recebendo denominação de N.I. (não identificados).

Posteriormente, os dados adquiridos foram catalogados e organizados de acordo com as famílias identificadas, que em seguida foram analisadas estatisticamente com auxílio do programa 
ANAFAU, para cálculo dos índices biológicos (Moraes et al, 2003).

\section{Resultados e Discussões}

Foram capturados 760 indivíduos, distribuídos em 16 famílias. Dolichopodidae e Phoridae tiveram maior representatividade, apresentando frequências de 45,66\% e 32,11\% dos indivíduos respectivamente (Tabela 1). As famílias Heleomyzidae $(9,61 \%)$, Culicidae $(3,82 \%)$, Tachinidae $(2,63 \%)$, Muscidae (2,37\%) Calliphoridae (0,66\%), Cecidomyiidae (0,66\%), Psychodidae (0,66\%), Asilidae (0,26\%), Rhagionidae (0,26\%), Stratyomidae (0,26\%), Tabanidae (0,26\%), Asteiidae $(0,13 \%)$, Bombyliidae $(0,13 \%)$ e Mydidae $(0,13 \%)$ vieram em seguida, totalizando juntas cerca de $21,84 \%$ dos insetos coletados, além de, $0,39 \%$ não foram identificados.

Observou-se que entre as 16 famílias encontradas apenas três foram classificadas como super dominante, super abundante e super frequente, destacaram-se Dolichopodidae, Phoridae e Heleomyzidae com 664 registro de indivíduos. Classificadas como dominante, muito abundante e muito frequente as familias Culicidae, Muscidae e Tachinidae, essas 6 famílias foram consideradas predominantes e bioindicadores. As demais foram classificadas como não dominantes, comuns e variaram entre frequente e pouco frequentes.

As famílias super dominantes Dolichopodidae, Phoridae e Heleomyzidae constituem-se na maioria como decompositores e parasitas. A família Dolichopodidae tem seus adultos como predadores de insetos menores, a exemplo de larvas do gênero Medetera, que vivem em cascas de árvores e predam algumas espécies de coleobrocas (coleóptera: Curculionidae). Os Phorídeos, facilmente diferenciados devido a aspectos morfológicos externos característicos como presença de corcunda, quando adultos são comuns em diversos habitats, mas especialmente próximos a vegetações em decomposição. Suas larvas variam quanto ao hábito, podendo viver em materiais em decomposição como vegetais e animais ou até em fungos e outros insetos, agindo como parasitas, a exemplo de parasitismo em formigueiros. Por fim, os Heleomizídeos, que se encontram normalmente associados a locais úmidos e sombreados. As larvas geralmente alimentam-se de material em decomposição ou fungos (Triplehorn \& Johnson, 2015).

Tabela 1. Número de indivíduos, frequência e índices biológicos obtidos através de famílias da Dipterofauna associados a cultivos comerciais de coco, Santa Izabel do Pará-Pa, 2017.

\begin{tabular}{ccccccc}
\hline Família & № de indivíduos & Frequência $(\%)$ & Domin & Abund & Freq & Const \\
\hline Asilidae & 2 & 0,26 & ND & d & PF & W \\
\hline
\end{tabular}




\begin{tabular}{|c|c|c|c|c|c|c|}
\hline Asteiidae & 1 & 0,13 & ND & $d$ & $\mathrm{PF}$ & W \\
\hline Bombyliidae & 1 & 0,13 & ND & $d$ & $\mathrm{PF}$ & W \\
\hline Calliphoridae & 5 & 0,66 & ND & c & $\mathrm{F}$ & W \\
\hline Cecidomyiidae & 5 & 0,66 & ND & c & $\mathrm{F}$ & w \\
\hline *Culicidae & 29 & 3,82 & $\mathrm{D}$ & $\mathrm{ma}$ & MF & w \\
\hline *Dolichopodidae & 347 & 45,66 & SD & sa & $\mathrm{SF}$ & W \\
\hline *Heleomyzidae & 73 & 9,61 & SD & sa & SF & w \\
\hline *Muscidae & 18 & 2,37 & $\mathrm{D}$ & ma & MF & W \\
\hline Mydidae & 1 & 0,13 & ND & $d$ & $\mathrm{PF}$ & w \\
\hline *Phoridae & 244 & 32,11 & SD & sa & SF & w \\
\hline Psychodidae & 5 & 0,66 & ND & c & $\mathrm{F}$ & w \\
\hline Rhagionidae & 2 & 0,26 & ND & $d$ & $\mathrm{PF}$ & w \\
\hline Stratyomidae & 2 & 0,26 & ND & $d$ & $\mathrm{PF}$ & w \\
\hline Tabanidae & 2 & 0,26 & ND & $d$ & $\mathrm{PF}$ & w \\
\hline *Tachinidae & 20 & 2,63 & $\mathrm{D}$ & $\mathrm{ma}$ & MF & w \\
\hline N.I. & 3 & 0,39 & ND & $c$ & $\mathrm{~F}$ & W \\
\hline $\begin{array}{ll}\text { Total } & \text { de } \\
\text { indivíduos } & \end{array}$ & 760 & 100 & & & & \\
\hline
\end{tabular}

Domin=Dominância; (D) dominante; (ND) não dominante. Abund=abundância; (ma) muito abundante; (c) comum; (d) dispersa; (r) rara. Freq=frequência; (MF) muito frequente; (F) frequente; (PF) pouco frequente; Const=Constância; (W) constante; Pred=Predominância; $(\mathrm{P})$ predominante; (NP) não predominante ( N.I.) = Não identificados. *espécies predominantes (indicadores).

Conforme o índice de Shannon-Wiener, que determina a diversidade de espécies no local de estudo, tem-se o resultado de 2,0375, que demonstra que a família Dolichopodidae possui ampla representatividade do geral de indivíduos coletados. No índice de equitabilidade o resultado foi de 0,7721, mostrando assim que houve uniformidade entre os indivíduos, pois não ultrapassou o índice de 1 . O índice de riqueza de espécies, Margalef, apresentou o valor de 2,8482, considerado baixo, o que pode de certa forma ser explicado pelo fato da análise representar apenas uma ordem de insetos (Tabela 2).

Tabela 2. Índices biológicos da comunidade de dipteros presentes na cocoicultura paraense, município de Santa Isabel do Pará-Pa.

\begin{tabular}{ccc}
\hline Índice de Shannon $\left(H^{\prime} \neq 1 C\right)$ & Índice de riqueza (Margalef) & Índice de Equitabilidade \\
2,0375 & 2,8482 & 0,7721 \\
\hline Intervalo de Confiança de $\mathrm{H}(\mathrm{P}=0,05)=>[2.017118 ; 2.057921]$
\end{tabular}

\section{Conclusões}

O passo inicial para um sistema agrícola racional moderno é a adoção de tecnologias de manejo que se se adequem ao meio ambiente e que atinja o objetivo principal, que é chegar próximo 
à máxima produtividade. E através dos resultados da análise entomofaunística realizada no presente trabalho, podemos concluir que a maior parte dos insetos da ordem díptera encontrados em plantios comerciais de coco são de grupos funcionais benéficos das famílias Dolichopodidae, Phoridae Heleomyzidae, Culicidae, Tachinidae, Muscidae, Calliphoridae, Cecidomyiidae, Psychodidae, Asilidae,Rhagionidae, Stratyomidae, Tabanidae, Asteiidae, Bombyliidae e Mydidae, considerados inimigos naturais e detritívoros, os quais contribuem positivamente para a redução do estresse ambiental causado pela fruticultura intensiva.

\section{Referências}

CAVALCANTE, L. V. A nova geografia da produção de coco no brasil. 2015. Disponível em: <http://www.enanpege.ggf.br/2015/anais/arquivos/9/262.pdf>. Acesso em: 11 out. 2017.

FERREIRA, J. M. S.; FILHO, M. M. Profução Integrada de coco: Praticas fitossanitárias. Embrapa. Aracaju, SE. 107p. 2002.

GULLAN P. J.; CRANSTON, P. S. Insetos: Fundamentos da Entomologia. $5^{\text {a }}$ ed. Rio de Janeiro, 2017.

MARCICANO M. D. L.; NIHE S. S.; LIMA I. M. M. First Host Record for Winthemia analis (Macquart) (Diptera: Tachinidae: Exoristinae) in Brazil: Brassolis sophorae laurentii Stichel (Lepidoptera: Nymphalidae: Brassolinae). Neotropical Entomology. Nota científica. 38(4):550552. 2009.

MARTINS, C. R.; JÚNIOR, L. A. J. Evolução da produção de coco no Brasil e o comércio internacional - Panorama 2010. Aracaju: Embrapa Tabuleiros Costeiros, 2011. (Embrapa Tabuleiros Costeiros. Documentos, 164).

MORAES, R. C. B.; HADDAD, M. L.; SILVEIRA NETO, S.; REYES, A. E. L. Software para análise faunística. In: $8^{\circ}$ Simpósio de controle biológico. 2003, S. Pedro, SP. Anais do $8^{\circ}$ Siconbiol, v.1, n.1, p. 195.

SEMA. Classificação Climática do Pará Método de Köppen. 2017. Disponível em: <www.sema.pa.gov.br/download/classificacao_climatica_do_para.doc>.Acesso em 10 out. 2017.

SILVEIRA NETO S.; NAKANO O.; BARBIN D.; VILLA NOVA N. A. Manual de ecologia dos insetos. Piracicaba: Ceres; 1976.

TRIPLEHORN, C. A. \& JOHNSON, N. F. Estudo dos insetos. Tradução da $7^{a}$ edição de Borror and Delong's introduction to the study of insects. $2^{\mathrm{a}}$ ed., São Paulo: Cengage Learning, 2015. 809 p. 